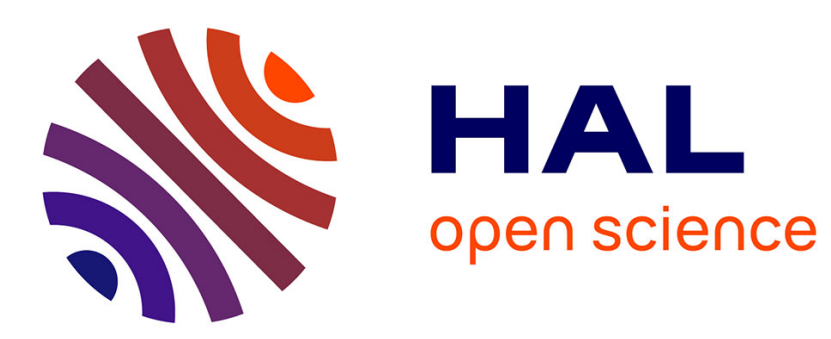

\title{
Semantic BMS: Ontology for Analysis of Building Automation Systems Data
}

\author{
Adam Kučera, Tomáš Pitner
}

\section{To cite this version:}

Adam Kučera, Tomáš Pitner. Semantic BMS: Ontology for Analysis of Building Automation Systems Data. 7th Doctoral Conference on Computing, Electrical and Industrial Systems (DoCEIS), Apr 2016, Costa de Caparica, Portugal. pp.46-53, 10.1007/978-3-319-31165-4_5 . hal-01438285

\section{HAL Id: hal-01438285 \\ https://hal.inria.fr/hal-01438285}

Submitted on 17 Jan 2017

HAL is a multi-disciplinary open access archive for the deposit and dissemination of scientific research documents, whether they are published or not. The documents may come from teaching and research institutions in France or abroad, or from public or private research centers.
L'archive ouverte pluridisciplinaire HAL, est destinée au dépôt et à la diffusion de documents scientifiques de niveau recherche, publiés ou non, émanant des établissements d'enseignement et de recherche français ou étrangers, des laboratoires publics ou privés. 


\title{
Semantic BMS: Ontology for Analysis of Building Automation Systems Data
}

\author{
Adam Kučera ${ }^{1}$, Tomáš Pitner ${ }^{1}$ \\ ${ }^{1}$ Masaryk University, Faculty of Informatics, \\ Laboratory of Software Architectures and Information Systems, \\ Botanická 68a, 602 00, Brno, Czech Republic \\ \{akucera, tomp\}@mail.muni.cz
}

\begin{abstract}
Building construction has gone through significant change with the emerging spread of ICT during last decades. "Intelligent buildings" are equipped with building automation systems (BAS) that can be remotely controlled and programmed and that are able to communicate and collaborate. However, BAS aim to facilitate operation of the building and do not provide sufficient support for strategic level decision support. This article presents adaptation of Semantic Sensor Network ontology for use in the field of building operation analysis. The Semantic BMS ontology enriches the SSN with model of building automation datapoints that gather operation data and describe the interconnections between BAS devices, algorithms and influenced or monitored properties of a building. Proposed ontology allows facility managers to query BAS systems in a way that is convenient for tactical and strategic level planning and that is unavailable in current state of the art systems.
\end{abstract}

Keywords: computer-aided facility management, building management systems, intelligent buildings, building automation systems, semantic web, ontology, semantic sensor network ontology, data integration

\section{Introduction}

Facility management is defined by the International Facility Management Association (IFMA) in following words: "Facility management is a profession that encompasses multiple disciplines to ensure functionality of the built environment by integrating people, place, process and technology."

We can distinguish several systems and/or data sources that can be utilized in order to support and simplify tasks of facility management staff, namely Building Information Model (BIM), Computer Aided Facility Management (CAFM) and Building Automation/Management Systems (BAS/BMS).

On the strategic level, CAFM systems are used for benchmarking of building operation efficiency and performance. Benchmarking methods in facility management are covered in EN 15221-7 standard. Requirements placed on Key Performance

\footnotetext{
${ }^{1}$ Available from http://ifma.org/about/whatis-facility-management
} 
Indicators (KPIs) are summarized in [1]. Among others, the authors mention flexibility, the quantitative nature of the KPIs and simplicity of use. BMS data satisfy the first three requirements, the simplicity of use is a downside of current BMS/BAS solutions. The paper proposes ontology description of the BAS data that largely facilitates use of the BAS data on a strategic level.

The paper is organized as follows: Section 2 describes contribution of the research to the field of cyber-physical systems. Section 3 presents state of the art in the field of semantic technologies for building automation. Section 4 describes the basic concept of Semantic BMS ontology. Section 5 demonstrates the capabilities of the proposed ontology on sample SPARQL queries. The last section discusses the advantages of the presented work and proposes future research topics.

\section{Relationship to Cyber-Physical Systems}

Modern ("intelligent") buildings are equipped with variety of sensors and controllable devices (e. g. HVAC, security systems). Devices are integrated into the Building Automation System (BAS), also referred as Building Management System (BMS). Devices incorporated in BAS can be remotely controlled, monitored and queried. Actions performed in an user interface of BMS have direct impact on the physical world (e.g. opening a valve) and changes in the physical world are reflected by automation algorithms (e. g. air conditioning units adjusts operating parameters in response to changes of outdoor air temperature). BAS systems thus can be considered cyber-physical systems.

The BMS contains large amount of a precise, up-to-date and detailed data that are valuable for a building operation analysis and cannot be obtained any other way. Individual information objects (such as current temperature in particular room measured by a sensor) accessible in the BAS network are referred to as "datapoints" further in the paper.

Absence of structured semantic information prevents efficient querying of the data points for analytical purposes, as it is not possible to select and filter the data based on criteria such as type of source device, location of a measurement or measured quantity kind. If the data from particular data points are required (e. g. electricity consumption for the last month for each of the buildings on the site for comparison), the operators of the system have to manually gather the data point addresses by inspecting the building plans or user interface of the BAS.

The above mentioned problem clearly emerges when operating large BAS system. Masaryk University utilizes BAS network consisting of approximately 1500 devices communicating using BACnet automation protocol with hundreds of thousands data points available. The network covers 35 buildings with overall area of $120000 \mathrm{~m}^{2}$ at the site of University Campus Bohunice in Brno, Czech Republic and several more over the whole Brno city. The requirements of effective operation of large-scale installations of automation technologies are discussed in [8].

This paper presents Semantic BMS ontology that aims to provide novel semantic description of the building automation systems. This allows developers of business intelligence applications to effectively query the model and easily gather required 
building operation data based on parameters unavailable in current semantic description of building automation systems. Such parameters describe relation of measured data with the physical world and objects described by different information systems.

\section{Related Work}

Standardized building automation protocols such as BACnet (ISO 16484-5), LONWorks (ISO 14908-1), KNX (ISO 14543), or ZigBee generally cover operation of building automation devices, providing specifications for physical communication layer, data link and networking layer and application layer on the highest level. A specific setup of automation network is however unique to each installation site. Automation protocols focus on communication interfaces and do not provide tools for complex and structured description of datapoint semantics.

Advanced ontological representations of building automation systems pursue various goals. Overview of different approaches and aims in the field of building automation ontologies follows.

The concept of "Semantic Agents" ensuring different aspects of building operation (Energy management, Safety, Security, Comfort) is proposed in [2]. Semantic agents are complex applications facilitating semantically described automation data.

In [6], authors define several basic “ontology modules", addressing different aspects of automation systems' semantic description. In [10], the W3C Semantic Sensor Network ontology (see section 4 for further details) is extended by model of physical processes occurring in the building (e.g. adjacent room exchanging energy) in order to provide tool for building operation diagnosis and anomaly detection.

Concept of Building as a Service (BaaS) is introduced in [4], aiming to simplify development and maintenance of building automation installations. In [3], discovery services for smart building are proposed using enriched SSN ontology, however they lack structures needed for complex querying (e.g. hierarchy of locations). Instead, they aim to facilitate development of self-adapting control algorithms. Integration of different automation systems facilitated by ontological generic application model is proposed in [11]. A generic application model enables deployment of platformindependent system configuration to physical devices implementing different automation protocols. In [5], ontology for integration of different BAS is proposed. The ontology describes platform-independent "parameters" (data points) which can be observed or controlled.

The Linked Open Data approach for building automation data streams is facilitated by EDWH Ontology proposed in [9]. The aim of the EDWH ontology is to provide bridge between the SSN ontology and the W3C RDF Data Cube vocabulary, as the data are meant to be analyzed by OLAP data cube techniques. In [7], the authors use SSN-based ontology for energy management based on sensor data. The semantically described BAS data help to establish situation awareness at the strategy level, allowing multi-level evaluation of energy consumption (from organization level to the level of individual appliances). 
In general, existing ontologies either aims to describe aspects of BAS different from data analysis, use proprietary/ad-hoc structures for storing semantic data, ignore integration with BIM systems or do not provide domain-specific mapping of BAS systems to the SSN ontology.

\section{Semantic BMS Ontology}

Goal of the presented research is to provide a BAS-protocol-independent model of intelligent building systems. The proposed ontology aims to represent information (data) available for operation analysis. The Building Automation System can be viewed as a sensor and actuator network for the purposes of data analysis. Semantic description of sensor networks is a subject of extensive research, resulting in frameworks and tools such as SensorML language, Observations \& Measurements (O\&M) model or Semantic Sensor Network ontology (SSN). However, for the use in the domain of building automation, particular differences have to be taken into account, and SSN was thus extended into the Semantic BMS ontology (SBMS).

A semantic description of the BMS is not required to contain some information that would be duplicate (copy) of data available in the BAS, BIM or CAFM systems. The aim of the presented research is to enrich the BAS/BMS with semantic links to entities present in other systems (BIM, CAFM) and add new layer of semantic metadata that are not available elsewhere. However, in some cases replication of the information is convenient for effective querying.

The SBMS represents spatial relations within the built environment as a tree hierarchy ("site-building-floor-room"). Devices and device types taking part in building automation are modeled as well. This simplified model has to be provided by the BIM system. The ontology repository duplicates BIM data and is updated whenever source data change. This approach was chosen because it allows for efficient retrieval of frequent queries ("get all temperature sensors from the first floor of the building B2") and keeps the semantic model and BIM/OWL translation relatively simple.

The key concepts for accurate semantic annotation of sensor/actuator data are "Observed property" (OP) and "Feature of Interest" (FoI). The FoI represents an object of measurement. The OP represents specific information that we observe. In the domain of building automation, we can demonstrate the concepts on examples such as energy consumption (OP) of a specific building (FoI) or speed (OP) of a specific fan (FoI).The Semantic BMS further specializes the concepts. Namely, it restrict class and property definitions so as valid FoI can be either a device (e.g. valve, pump, engine, or PLC) or a location (site, building, floor, or room). The FoI has to be present in the BIM system.

The building operation data are generally not meant to be publicly available on the Internet. Therefore, there is no need for semantic annotation of individual measurements. For that reason, the SSN Observation concept represents only semantic connection of datapoint, sensor, FoI and OP and do not contains any actual measured data. Annotated data point values can then be directly obtained from the BAS. 
The Semantic BMS Ontology introduces simplified model of processes occurring in the BAS system as an association of input and output -- room temperature serves as an input for regulation algorithm of a respective AC unit, thus influencing values of various datapoints that the algorithm controls (e. g. fan speed). Furthermore, the ontology describes "indirect" influence observed as a result of physical processes occurring in the built environment. The indirect influence usually occurs between output of one algorithm and input of another. As an example, we can consider a datapoint A representing openness of a valve that mixes cold and hot water. The state of the valve is controlled by a datapoint acting as an output of an algorithm. Different control algorithm uses as an input a datapoint B representing water temperature past the valve. Thus, the datapoint A value indirectly influences the datapoint B value -the measured temperature depends on the state of the valve.

The ontology further specifies domain-specific sensing methods, provides categorization of observed physical qualities and provides model of generic data point types.

\section{Results}

At the moment, the Semantic BMS ontology is implemented using OWL language and populated with sample data imitating relationships in real facilities. ${ }^{2}$ The Semantic BMS ontology defines 107 classes ( 79 of them however represent device types and are adapted from Industry Foundation Classes 4 standard) and 34 relations (properties). In most of the cases for both classes and relations, the added elements are specializations of existing SSN elements. For testing purposes, the ontology is populated with 175 individuals (e.g. buildings, rooms, devices, datapoints) and their respective relations.

The key classes are named Site, Building, Floor, Room, and Device, representing BIM elements, Address (representing various types of datapoints) and several classes extending original definitions from the SSN ontology: Observation, Scope (representing Feature of Interest), Observed property and Sensing.

As an ontology repository, Apache Jena TDB is used. The repository can be queried using SPARQL query language and allows to select and filter data points according to their relation to physical world and to entities described by a BIM system. Following SPARQL query extracts available information about particular data point (PREFIX declarations are omitted):

SPARQL Query: Description of a data point

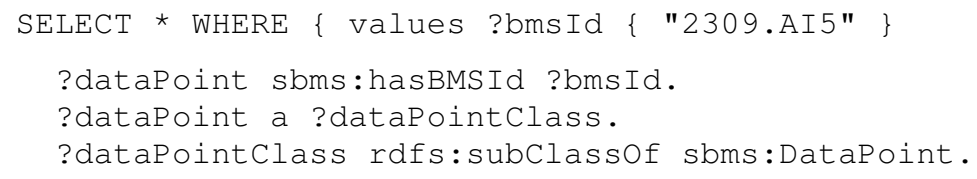

${ }^{2}$ Available from http://is.muni.cz/www/255658/sbms/v1_0/?lang=en 


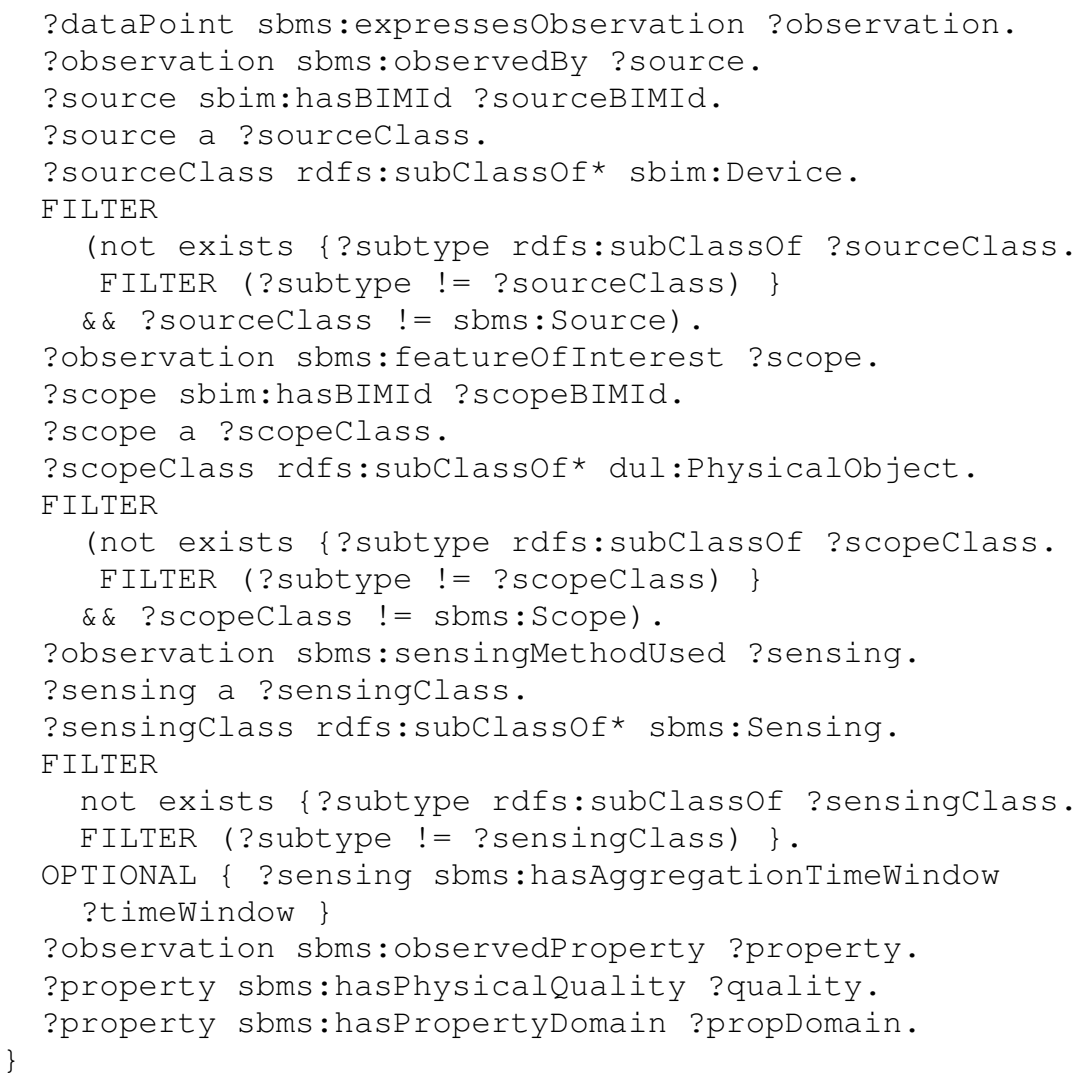

The presented query provides following information about the respective datapoint with BACnet address of "2309.AI5":

- Datapoint type - Specifies a role of the datapoint. Possible types can be Input, Output, User defined value, Auxiliary, Algorithm and Historical data.

- Data source - Specifies a device (further described in the BIM database) that provides the BAS with the data.

- $\quad$ Source device class - Describes type of the source device (e.g. Energy meter). The list of device types is derived from the IFC 4 specification.

- Scope (Feature of Interest) - A scope specifies an object the datapoint value is related to (e.g. fan in the case of an data point representing fan speed, room when measuring room temperature or building when measuring energy consumption).

- $\quad$ Scope type - Specifies if the scope is a location or a device.

- Physical quality (Observed Property) - Specifies (usually) physical quality represented by the datapoint (e.g. temperature, humidity, energy, or output power). The list of available qualities is based on the Unified Code of Units of Measure (UCUM) and can be extended to meet specific requirements. 
- $\quad$ Sensing method and time window - Different data point implement different sensing methods. The simplest case is direct sensing (e.g. room temperature). Other (indirect) sensing methods employ some kind of aggregation over time period (e.g. energy consumption total for the last month).

Described parameters allow facility managers to query the BAS data storage in a way unavailable in the BAS systems themselves. The SBMS facilitates queries based on an origin of the data and their role in the building operation. Additionally, a query can inspect relations between data points and influenced operational parameters of the building using simplified model of processes.

However, facility managers should not be forced to learn SPARQL language nor to construct complex queries by themselves. The queries are meant to be constructed by an API available as a part of a middleware layer described in the following section. The API then will be used by applications for end users equipped with convenient user interface for data selection and visualization.

\section{Conclusions and Future Work}

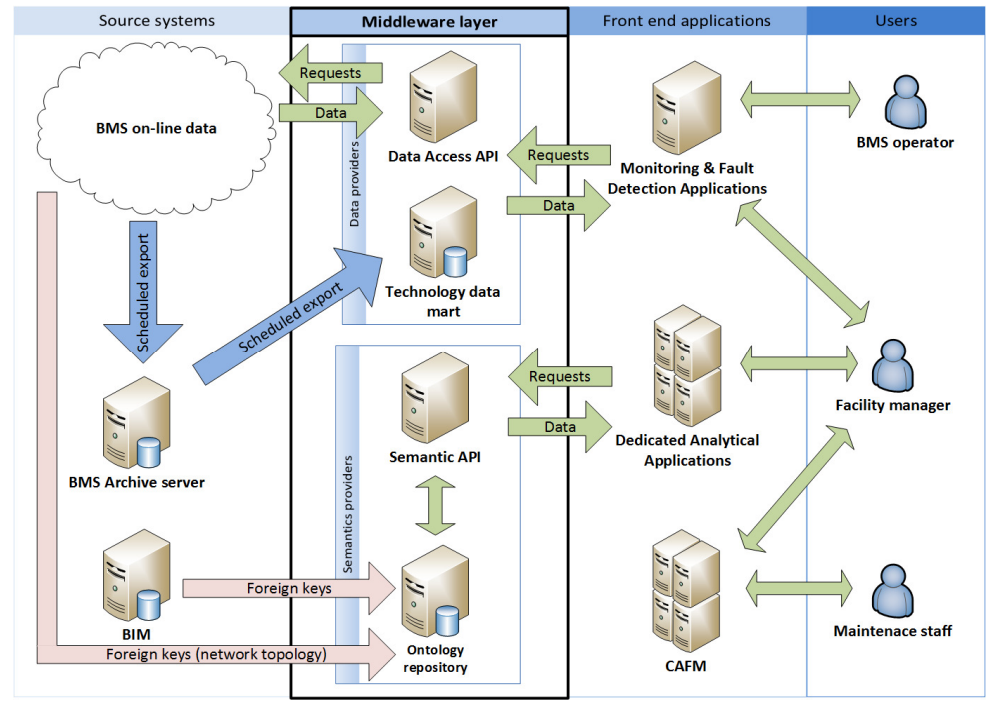

Fig. 1. Semantic BMS middleware.

The proposed ontology introduces novel approach to querying building automation cyber-physical systems. Querying is based on relations of datapoints with the physical world, contrary to common approach based on network topology based querying. The proposed method allows flexible querying for strategic level decision support in the field of facility management. 
Future work includes development of middleware layer providing RESTful APIs providing convenient methods both for semantic querying and data access (see Fig. 1). The APIs will be designed and developed with respect to the typical building performance analysis queries, mainly based on benchmark indicators proposed in "EN 15221-7 Benchmarking in Facility Management" standard.

Other challenges include effective methods to populate the ontology with semantic data and on seamless integration of the Semantic BMS ontology with BIM data sources by providing explicit mapping between Semantic BMS ontology concepts and objects described by Industry Foundation Classes standard used for data exchange in BIM systems.

\section{References}

1. Alwaer, H., Clements-Croome, D.J.: Key performance indicators (KPIs) and priority setting in using the multi-attribute approach for assessing sustainable intelligent buildings. Building and Environment 45(4), 799-807 (Apr 2010)

2. Andrushevich, A., Staub, M., Kistler, R., Klapproth, A.: Towards semantic buildings: Goaldriven approach for building automation service allocation and control. In: 2010 IEEE Conference on Emerging Technologies and Factory Automation (ETFA). pp. 1-6 (Sep 2010)

3. Bovet, G., Ridi, A., Hennebert, J.: Toward Web Enhanced Building Automation Systems. In: Bessis, N., Dobre, C. (eds.) Big Data and Internet of Things: A Roadmap for Smart Environments, vol. 546, pp. 259-283. Springer International Publishing, Cham (2014)

4. Butzin, B., Golatowski, F., Niedermeier, C., Vicari, N., Wuchner, E.: A model based development approach for building automation systems. In: 2014 IEEE Emerging Technology and Factory Automation (ETFA). pp. 1-6 (Sep 2014)

5. Caffarel, J., Jie, S., Olloqui, J., Martnez, R., Santamara, A.: Implementation of a Building Automation System Based on Semantic Modeling. Journal of Universal Computer Science 19(17), 2543-2558 (Nov 2013)

6. Christoph, L.: Semantics to the Shop Floor: Towards Ontology Modularization and Reuse in the Automation Domain. pp. 3444-3449 (Aug 2014)

7. Curry, E., Hasan, S., O'Riain, S.: Enterprise energy management using a linked dataspace for Energy Intelligence. In: Sustainable Internet and ICT for Sustainability (SustainIT), 2012. pp. 1-6 (2012)

8. Kučera, A., Pitner, T.: Intelligent facility management for sustainability and risk management. In: Hřebíček, J., Schimak, G., Kubásek, M., Rizzoli, A. (eds.) Environmental Software Systems. Fostering Information Sharing. pp. 608-617. Springer, Berlin Heidelberg (2013)

9. Mehdi, M., Sahay, R., Derguech, W., Curry, E.: On-the-fly Generation of Multidimensional Data Cubes for Web of Things. In: Proceedings of the 17th International Database Engineering \& Applications Symposium. pp. 28-37. IDEAS '13, ACM, New York, NY, USA (2013)

10.Ploennigs, J., Schumann, A., Lecue, F.: Extending semantic sensor networks for automatically tackling smart building problems. In: 2014 European Conference on Artificial Intelligence (ECAI). pp. 1211-1214 (2014)

11.Reinisch, C., Granzer, W., Praus, F., Kastner, W.: Integration of heterogeneous building automation systems using ontologies. In: 34th Annual Conference of IEEE Industrial Electronics, 2008. IECON 2008. pp. 2736-2741 (Nov 2008) 